\title{
Integration of Brassinosteroid Signal Transduction with the Transcription Network for Fiber Development and Drought Stress in Gossypium hirsutum L
} Deepti Nigam*

Plant Molecular Biology and Genetic Engineering Laboratory, National Botanical Research Institute, Rana Pratap Marg, Lucknow, India

\begin{abstract}
Brassinosteroids (BRs) are a family of steroid hormones present ubiquitously in plant kingdoms and regulate wide range of physiological and developmental processes. BRs signal to regulate brassinosteroid transcription factors (BRTF's), which regulate target genes for various BR responses. However, the role of BRs in developing fiber and during drought stress which is major limiting factor in its development is still limited.

Our in silico modelling using Algorithm for the Reconstruction of Accurate Cellular Networks (ARACNe) reveals that BRTF's form a gene regulatory network (GRN) during cotton fiber development. Hierarchical clustering highlighted gene expression during acute dehydration response from early, peak and late elongation stage. SEA (Singular Enrichment Analysis) based gene ontology analysis demonstrate synergistic effect of BRs with other hormones like auxin. Our results provide a strong footprint of BRs based gene regulation in cotton fiber growth and drought stress.
\end{abstract}

Keywords: ARACNe Algorithm for the Reconstruction of Accurate Cellular Networks; GRN gene regulatory network; BRs Brassinosteroids; BRTF's Brassinosteroid Transcription Factor's; Gossypium hirsutum

\section{Introduction}

Cotton is the bulkiest sink of natural textile fiber and a main oilseed crop, with extensive scientific, environmental, and socio-economic footsteps. Cotton fiber is a highly elongated seed hair with a secondary wall composed of almost pure cellulose. Development of cotton fibers is positively affected by a number of phytohormones including auxin, gibberellins and brassinosteroids (BRs) [1,2]. BRs consist of a family of phytosterols found throughout the plant kingdom [3]. In general, $\mathrm{BR}$ has effects similar to auxin. BR stimulates germination, shoot elongation, vascular development and pollen formation. BR inhibits root elongation and promotes secondary root initiation. BR accelerates cell elongation and affects cytoskeletal and cell wall structure. BR promotes the transverse orientation of microtubules in azuki bean [4], and the brassinolide (BL)-induced epicotyl growth in Cicer arietinum is accompanied by increased $\beta$-tubulin expression [5]. Among the first BR-responsive genes to be identified, BR-unregulated gene 1 (BRU1) from soybean [6] was identified as a xyloglucan endotransglycosylase gene (i.e. XTH). These enzymes are involved in cell wall loosening [6] and provide a direct link between BR and its effects on cell wall development. Microarray analysis of BR-up-regulated genes showed that many cell wall-related genes, including fatty acid elongase (At1g01120), expansin (EXP), arabinogalactan protein (AGP), acyl carrier protein (ACP) and a number of XTHs (U43488,), are up-regulated by BL in Arabidopsis [7,8]. Interestingly, the cotton counterparts of these genes were also reported to be preferentially expressed in cotton fibers [9].

Cotton fiber development can be divided into four overlapping stages: fiber initiation, elongation, secondary wall deposition and maturation. Fiber elongation is controlled by the interaction of cell turgor and cell wall extensibility [10]. Allen et al. [2] reported that transgenic cotton that was BR-stimulated overexpress a cotton XTH known as KC22 and these plants produce fibers that are significantly longer than those from control plants [10].
Further, BES1 (BRI1-EMS-SUPPRESSOR 1) is a novel plantspecific transcription factors that accumulates in the nucleus in response to BRs, and plays a role in BR-regulated gene expression; however, the mechanism by which BES1 regulates gene expression in developing fiber cell is still unknown $[11,12]$. Our in silico identification of BRTF's target genes and their gene ontological process confirm that the expression of BR-responsive genes correlates with fiber development and drought stress also.

\section{Material and Methods \\ Data Collection and initial Processing}

108 cotton fiber microarray data sets were downloaded from Gene Expression Omnibus database (http://www.ncbi.nlm.nih.gov/geo/). Data are available at GEO data (Series GSE36021 and GSE29810). Data was of oligonucleotide data, and were usually provided in .cel file format. All available data were included in processing and analysis. All data sets were log transformed and median centered per array, and the standard deviations were normalized to one per array. Eight cotton BES1 genes (BRTF's) were selected from the Plant TFDB (http:// planttfdb.cbi.pku.edu.cn) for network analysis and their chip Ids were extracted from Plexdb tool (http://www.plexdb.org/). We have also taken the previously reported supplementary data concerning to BES1 transcription factor and their GRN regulation in Arabidopsis for validation, where similar type of study have performed for only one BES1 transcription factor [13].

*Corresponding author: Deepti Nigam, Plant Molecular Biology and Genetic Engineering Laboratory, National Botanical Research Institute, Rana Pratap Marg, Lucknow, India, Tel: +915222297914; E-mail: deept_mbi@yahoo.co.in

Received March 29, 2014; Accepted April 16, 2014; Published April 19, 2014

Citation: Nigam D (2014) Integration of Brassinosteroid Signal Transduction with the Transcription Network for Fiber Development and Drought Stress in Gossypium hirsutum L. J Comput Sci Syst Biol 7: 108-114. doi:10.4172/jcsb.1000145

Copyright: (c) 2014 Nigam D. This is an open-access article distributed under the terms of the Creative Commons Attribution License, which permits unrestricted use, distribution, and reproduction in any medium, provided the original author and source are credited. 
Citation: Nigam D (2014) Integration of Brassinosteroid Signal Transduction with the Transcription Network for Fiber Development and Drought Stress in Gossypium hirsutum L. J Comput Sci Syst Biol 7: 108-114. doi:10.4172/jcsb.1000145

\section{Network inference using ARACNe}

Using a combined stringent cut off of an error tolerance $\varepsilon=0.2$ and a p-value threshold of mutual information (MI) at 0.05 , ARACNE (Algorithm for the Reconstruction of Accurate Cellular Networks) (http://wiki.c2b2.columbia.edu/califanolab/index.php/Software/ ARACNE) inferred global gene networks for 8 brassinosteroid transcription factors genes (GhiAffx.23450.1.A1_s_at,Ghi.2138.1.S1_ at,Ghi.2429.1.S1_s_at,Ghi.8007.1.S1_at,Ghi.9165.1.S1_s_at, Ghi.9165.1.S1_s_at,GraAffx.19173.1.A1_s_at,Ghi.6198.3.A1_at, GraAffx.34276.1.A1_s_at). These genes were considered as a "hub genes" genes for target identification. ARACNe, was developed and successfully used to infer a GRN in human B cells [14,15]. If the TFs indeed function to mediate abiotic stress responses, we should be able to observe some expression correlation among them, which can be demonstrated by reconstruction of a gene regulatory network $(\mathrm{GRN})$. ARACNe inferred direct interactions ( $1^{\text {st }}$ neighbours) and $2^{\text {nd }}$ neighbours for selected hub genes. Threshold for MI (mutual information) and DPI (Data Processing Inequality) tolerance were set to $\mathrm{p}<0.05$ (Bonferroni corrected for multiple testing) and $0 \%$, respectively. The bootstrapping option was applied to generate 100 bootstrapped networks. These networks were merged into a consensus network from consensus voting methods based on a statistically significant number of interactions inferred from the bootstrapping steps. Further Cytoscape 3.0.2 software was used to draw the network using the same parameter as described in [16].

\section{Annotation analyses of Cotton Gene chip}

TF's Targets genes were analysed using the functional categorization based on three GO categories at $\mathrm{p}$-values $\geq 0.05$. The agriGO tool (http://bioinfo.cau.edu.cn/agriGO/) was used to perform the enrichment analysis using SEA (Singular Enrichment Analysis) coupled with available background data of cotton probes. Cotton Gene chip annotation was based on the top hits using the PLEXdb tool.

\section{Metabolic Interaction Network analysis}

The BRTF's target genes were analyzed for cellular pathway and transcriptome interactions using the program Pathway Studio (Ariadne Genomics, USA). The pathway diagram was further filtered to show the proteins involved in cellular processes associated with the fiber development network.

\section{Results}

\section{Brassinosteroid Regulatory gene networks}

Firstly we have taken BRTF's from cotton transcription factor database (http://www.bmicc.org/web/english/search/planttfdb) then we perform blastn with the sequences of cotton affymetrix chip where we found total 8 BES1 genes. (Figure 1 and Table 1). Using the ARACNe we found 3636 target genes for these regulatory genes which were further annotated for their ontology categorization.

To gain an insight over BRs regulated BRTF's we used the supplementary data for Arabidopsis thaliana BES1 target genes, BR and mutant bes-1 differentially regulated genes data provided by Xiaofei Yu et al. [12]. We performed the Venn analysis between our identified cotton BES1 target genes (i.e BRTF's) and putative Arabidopsis BES1 target genes (Figure 2) and BR differentially regulate (up/down) and bes $1-D$ mutant differentially regulated (up/down) genes (Figure $3 a$ and 3b) using Venny tool (http://bioinfogp.cnb.csic.es/tools/venny/). We selected top 46 genes as BR regulated BRTF's and were further used for expression profiling in drought effected developing fibers and in developing fibers of contrasting genotypes.

\section{Singular Enrichment Analysis of BRTF's target genes}

The 3636 target genes were further used for Fisher Exact test based Gene ontology enrichment analysis using agriGO tool (http://bioinfo. cau.edu.cn/agriGO/) where FDR-adjusted p-value of $\leq 0.05$ was

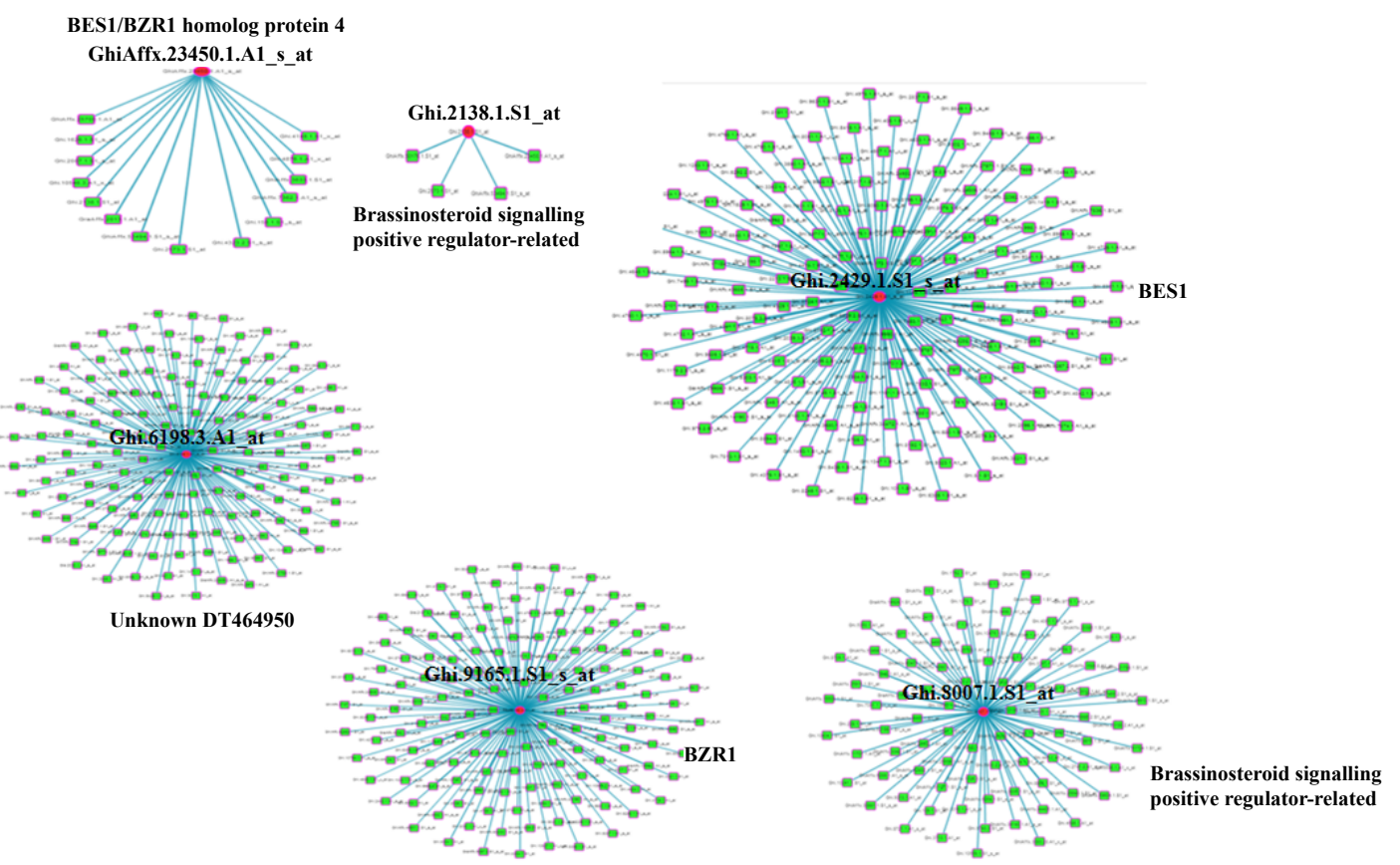

Figure 1: Interactome analysis showing brassinosteroid transcription factors (BRTF's) and their targets interaction using ARACNe algorithm. Red circle showed hub TF'S and green circles are their targets. 


\begin{tabular}{|c|c|}
\hline Selected Brassinosteroid Transcription factors i.e. "Hub Gene” & No of Targets \\
\hline Ghi.2429.1.S1_s_at & 1997 \\
\hline Ghi.6198.3.A1_at & 999 \\
\hline Ghi.9165.1.S1_s_at & 532 \\
\hline Ghi.8007.1.S1_at & 90 \\
\hline GhiAffx.23450.1.A1_s_at & 14 \\
\hline Ghi.2138.1.S1_at & 4 \\
\hline GraAffx.19173.1.A1_s_at & 0 \\
\hline GraAffx.34276.1.A1_s_at & 0 \\
\hline
\end{tabular}

Table 1: Regulatory Brassonosteroid transcription factors and their target genes identified through ARACNe algorithm. Only top six transcription factors and their targets network data were used for Cytoscape 3.0.2.

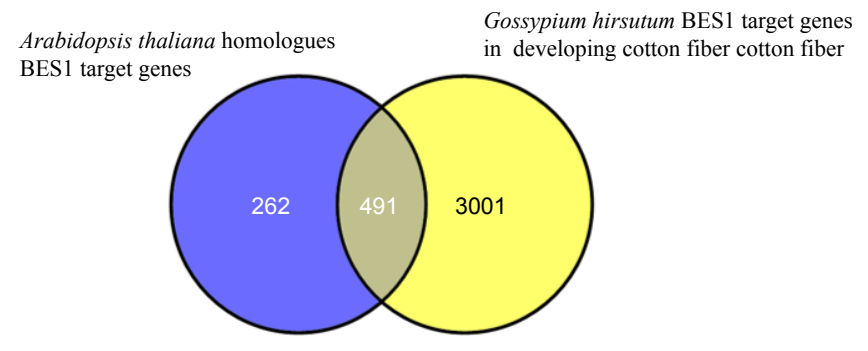

Figure 2: Venn analysis for overlapping BRTF's target genes of Gossypiumhirsutum and BES1 target genes of Arabidopsis thaliana.

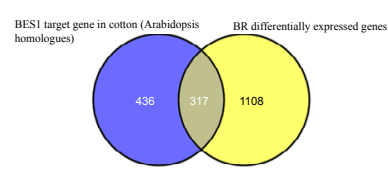

(a)

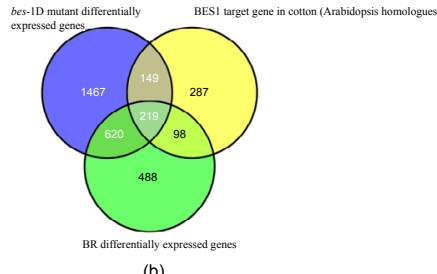

(b)
Figure 2: (a) Venn analysis for overlapping BES1 target gene and brassinosteroid differential genes in cotton (Arabidopsis homologues) (b) Venn analysis for overlapping gene in between BES1 target gene, bes-1D mutan differentially expressed genesand brassinosteroid differential genes in cotton (Arabidopsis homologues).

chosen as the cutoff (Figure 6, Supplementary file S1). The distribution of BRTF's target enriched GO terms showed several noteworthy findings. The significantly enriched GO terms, including response to stimulus (GO: 0050896, $p$-value $=4.10 \mathrm{E}-06$ ), transcription factor complex (GO: 0005667, $p$-value $=1.20 \mathrm{E}-05$ ), response to hormone stimulus (GO: 0009725, $p$-value $=2.30 \mathrm{E}-05$ cellular nitrogen compound metabolic process (GO: 0034641, $p$-value $=3.10 \mathrm{E}-05$ ), response to abiotic stimulus (GO: 0009628, $\mathrm{p}$-value $=0.0023$ ) and transcription regulator activity (GO: 0030528, $\mathrm{p}$-value $=5.30 \mathrm{E}-05$ ), response to brassinosteroid stimulus (GO: 0030528, p-value $=0.0024$ ), response to auxin stimulus process (GO: 0009733, $p$-value $=0.0034$ ).

\section{BRTF's target genes showed interesting pattern during} drought affected developing fiber

A of number of upstream pathways were activated during whole fiber developmental stages. Here we observed an interesting expression pattern of BRTF's target genes for drought effected developing fiber.
We obtained four different expression patterns for BRTF's target gene expression based on their peak expression (Figure 4). A number of studies have indicated that BRs are able to enhance the capability of plants to cope with stresses, such as heavy metal stress, water stress, and salt stress, high and low temperature stress and pathogen attack [1719]. We annotate target genes and found a total 42 TF's in these patterns that may be biologically relevant. Previous analysis revealed that $\mathrm{BR}$ induces the expression of heat shock protein (HSP83, HSP70, Hsc70-3, and $H s c 70-G 7)$, heat shock factor (HSF3), and oxidative stress-related genes (GST, ATPA2, and ATP24a) in Arabidopsis [8,20]. BR induces defense and antioxidant genes in the absence of stresses. In contrast, expressions of cold and pathogenesis-related genes are reduced in Brz-treated cucumber seedlings or BR-deficient Arabidopsis mutants $[20,21]$. These results suggest that BR enhances plant stress tolerance by activating genes involved in plant defense and stress responses. Further Pustovoitova et al. [22] observed that cucumber sprayed with a synthetic BR, 24-epibrassinolide (EBR), had improved resistance to dehydration. BR treatment has also been reported to increase germination and seedling growth of Sorghum vulgare under osmotic stress [23] and enhance drought tolerance in Phaseolus vulgaris [24].

\section{BRTF's target genes showed contrasting pattern in between the developing fiber of contrasting genotypes}

In our previous study we focused on the differential genes and metabolic pathways between superior (NBRI777 and NBRI725) and inferior genotypes (NBRI 703, NBRI783, NBRI737) [24]. Here we characterize transcriptional dynamics in contrasting cotton genotypes to elucidate time-dependent and genotype-specific modulation of fiber development which leads to difference in enhance fiber phenotype. Our present study both extended the comparison of their genome and tracks the expression of BRTF's targeted genes over a fiber developmental stage (Figure 5). Functional classification of the BRTF's targeted genes (BR-regulated genes excluding both high- and lowconfidence BZR1-binding targets) based on Gene Ontology categories showed that BR directly and indirectly regulates a range of biological processes and cellular activities (Figure 6). For example, genes involved in transcription, cell growth as well as microtubule associated complex are highly enriched in BRTF's targeted genes. Genes involved in solute cation/ or hydrogen symporter activity, sugar transport cell wall organization are equally enriched in BRTF's target genes. Nigam et al. [16] reported that BR activates a large number of BES1/BZR target genes encoding cell wall-related enzymes, such as cellulose synthase, pectinesterases, xyloglucosyl transferases, and expanins, which are likely to mediate BR promotion of fiber cell elongation and differentiation. $\mathrm{BR}$ also directly activates a large number of genes involved in cellular transport and cytoskeleton organization, which are likely to contribute to BR promotion of solute uptake and directional cell elongation. Similarly, we found enrichment of UDP-glycosyltransferase (UGT) in our target genes. This is also supported by one of the reports of Gilbert et al. [25] where the high accumulation of ABA in mutant fiber suggests a possible role of UGT73C14 for ABA homeostasis in the $L i$ mutant fibers. Higher expression levels of UGT73C14 in mutant $\mathrm{Li}_{2}$ fiber lines could likely be a feedback reaction to changed ABA levels, that probably acting to "detoxify" fiber cells by targeting excess ABA for storage.

\section{Metabolic Interaction Network showing functional dynam- ics of BRTF target genes during fiber development governing drought tolerance and superior fiber quality trait}

To interpret functional associations between co-expressed BRTF's and their target gene, we mapped the target genes using the Arabidopsis 


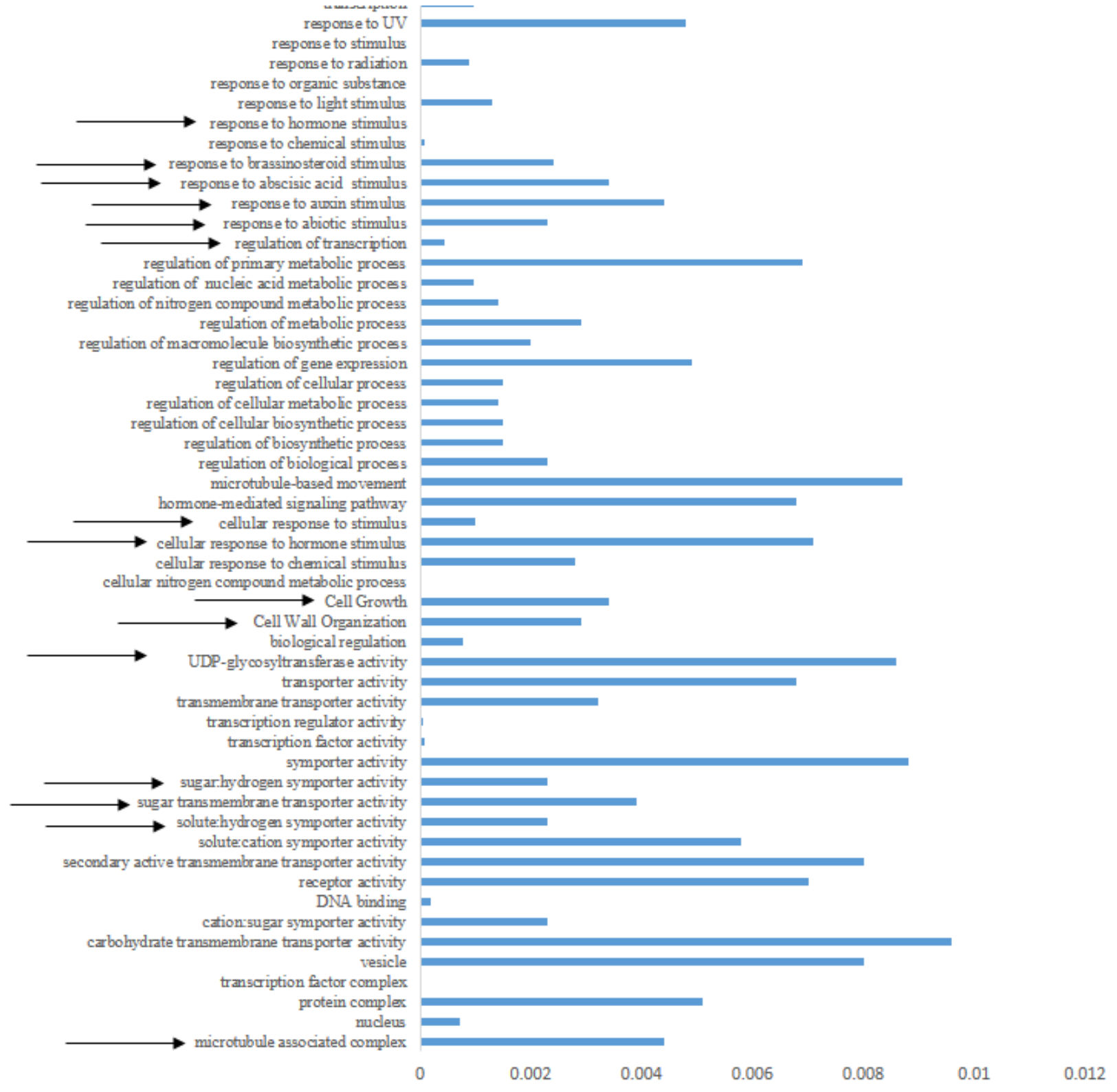

Figure 4: SEA analysis of BRTF's target genes showing enriched Biological, Molecular and Cellular Process.

thaliana locus ids and performed Interactome analysis using Pathway studio 5.9 software with a parameter of $\mathrm{p}$-value $\leq 0.05$. We observed that BRTF's target gene supports their role in a diversity of physiology processes within fiber cell (Figure 7). Analysis of the highly connected biological process includes cell division, cell proliferation, cell expansion differentiation, disease resistance, vacuole biogenesis etc. Whereas it was found that these genes also have interaction with other phytohormones like auxin, Jasmonic acid, ethylene which are well reported in having important role in developing fiber.

\section{Hypothetical Model for superior fiber quality genotypes less prone to drought stress}

We also observed the harmonious action of auxin and brassinosteroid biosynthetic pathway alterations along with change in expression of cell wall enzymes during switch from elongation to SCW in fiber cell of superior genotypes lead to better fiber quality in contrast to inferior. Further, we found GhBES1 to be highly expressed at 25 DPA. GhBES1 (BRI1-EMS-SUPPRESSOR 1) transcripts is known as positive regulator of brassinosteroid signaling [23]. Our gene ontology analysis result suggests the same phenomenon where we observed equal enrichment of brassinosteroid and auxin hormones (Figure 6). We found BRTF's target genes showed peak expression not only at the later stage in superior genotypes as in previous report but a strong and constitutive expression at all fiber developing stages (Figure 6). We have hypothesize a model where future functional analyses are required to test the many hypotheses generated here, and to explore their individual and synergistic effects on cellular physiology and ultimately, morphology of fiber (Figure 8).

\section{Conclusion}

Transcriptional regulation is pivotal for many hormones for effect 


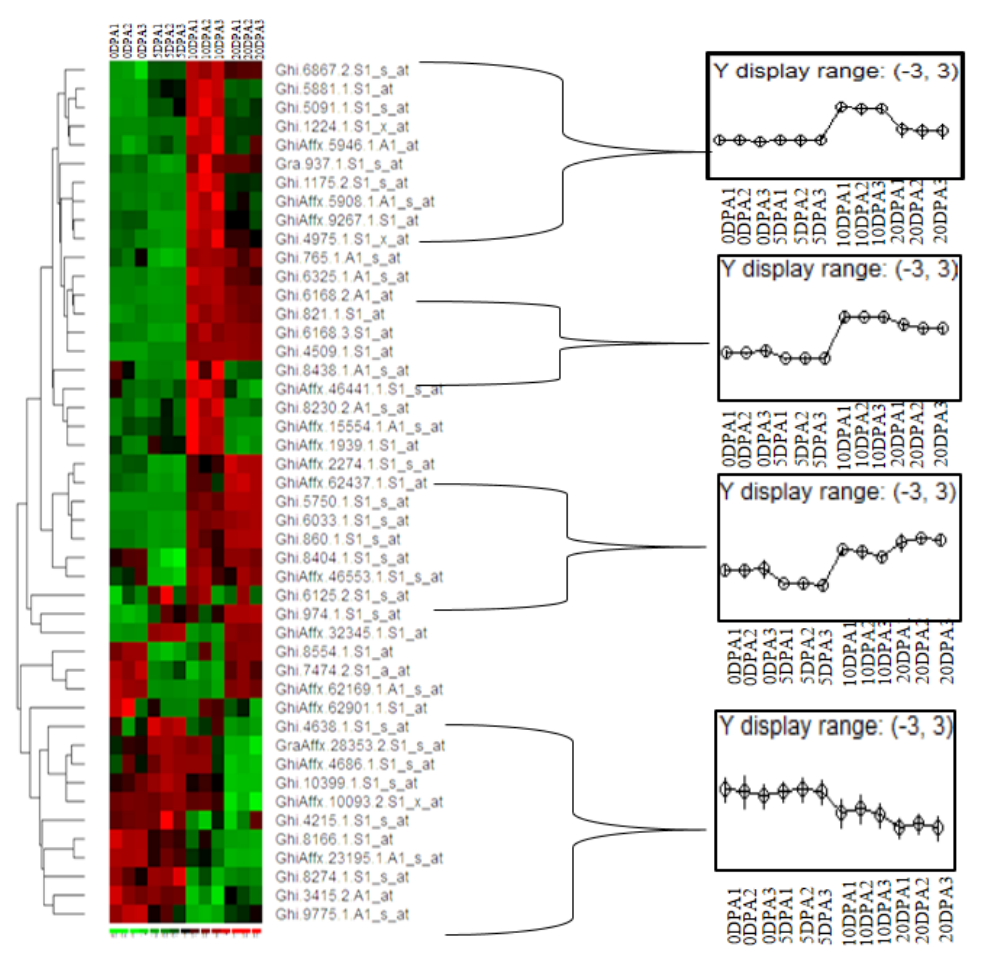

Figure 5: Expression pattern of BES1 target genes during different fiber development stages of Gossypiumhirsutumeffected with drought stress.
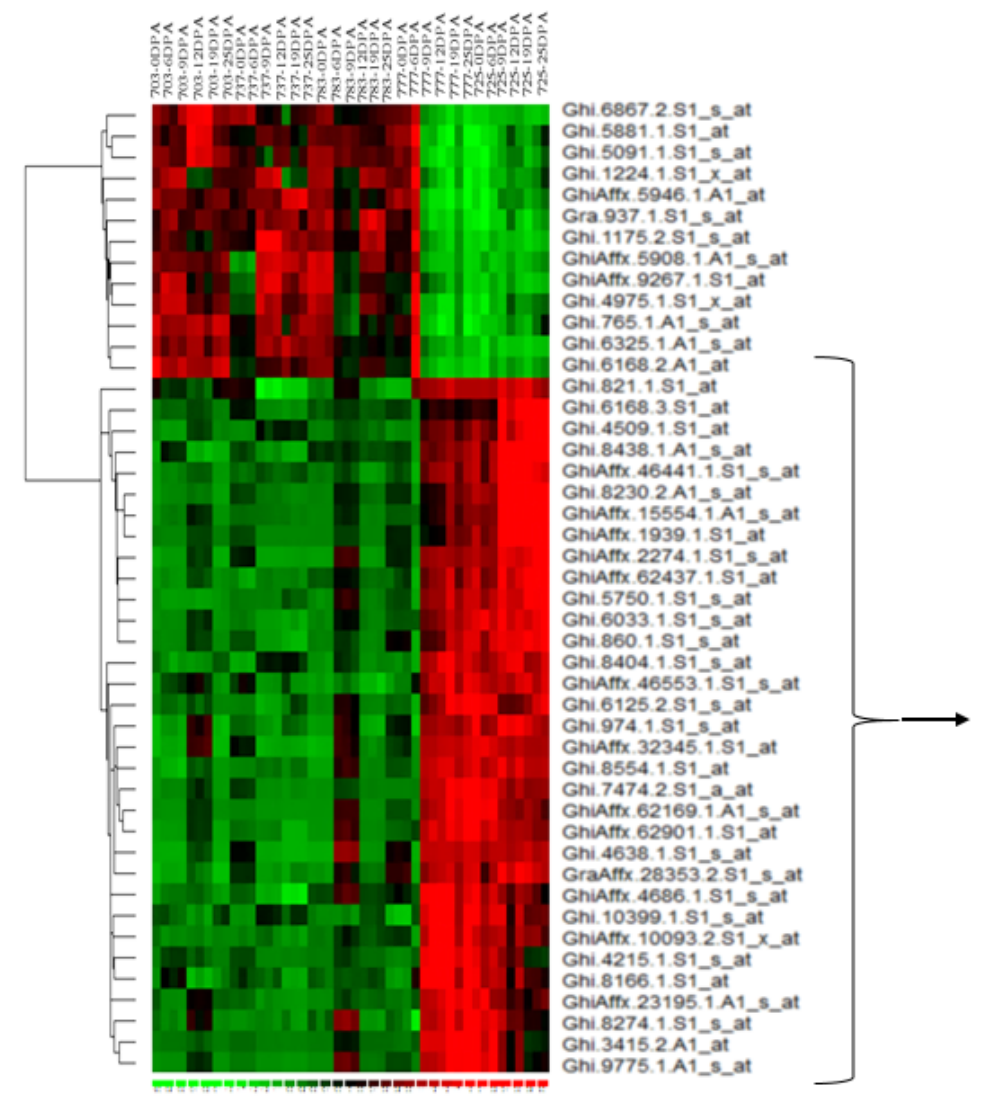

BRTF's target genes expression biasness within superior fiber quality genotypes

Figure 6: Expression of BES1 target genes during fiber development stages in contrasting genotypes of Gossypiumhirsutum. 
Citation: Nigam D (2014) Integration of Brassinosteroid Signal Transduction with the Transcription Network for Fiber Development and Drought Stress in Gossypium hirsutum L. J Comput Sci Syst Biol 7: 108-114. doi:10.4172/jcsb.1000145

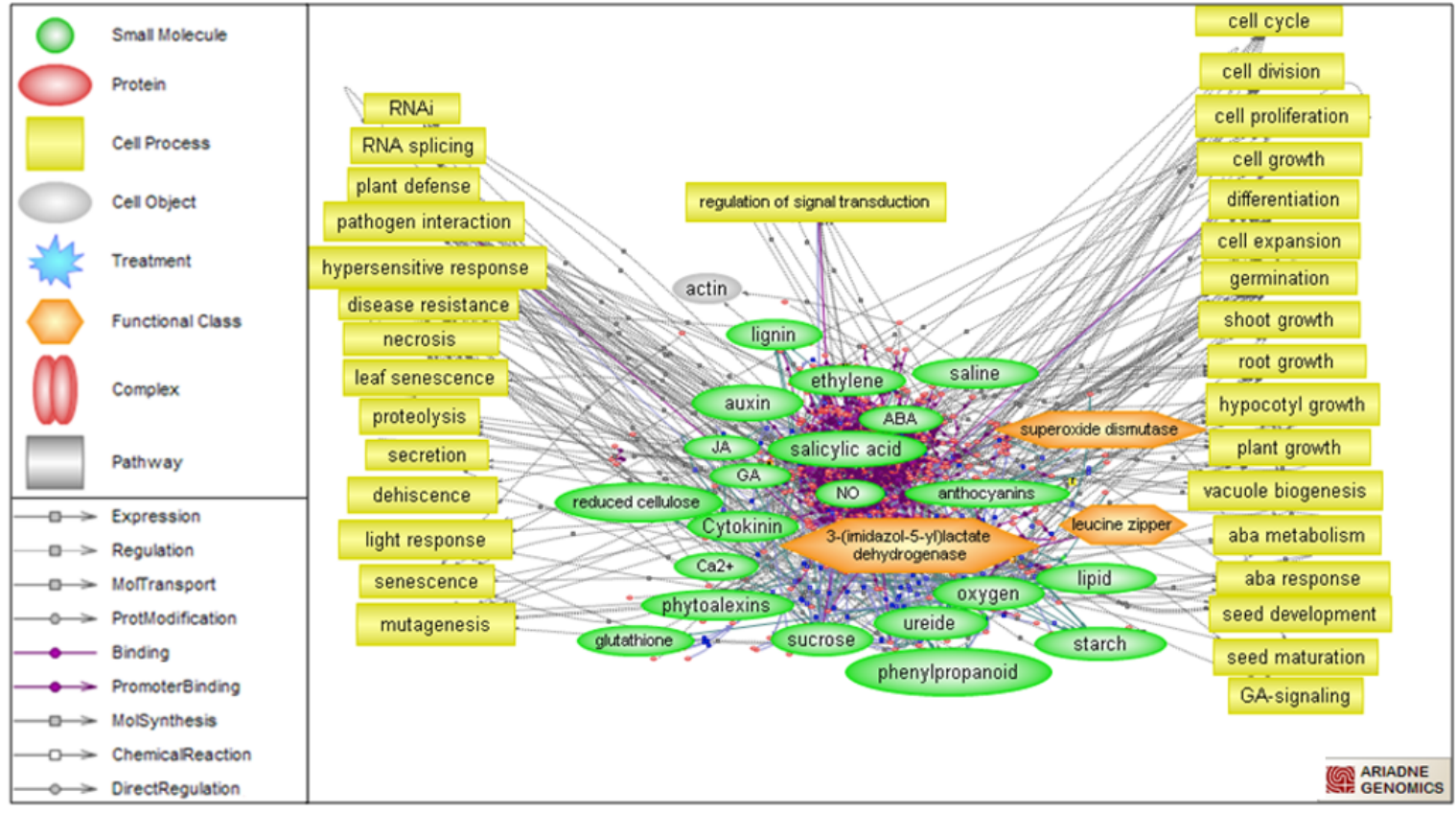

Figure 7: Metabolic pathway analysis of BRTF's target genes showing highly connected metabolic process probably leading to drought resistant and better fiber quality in superior genotypesof Gossypiumhirsutum.

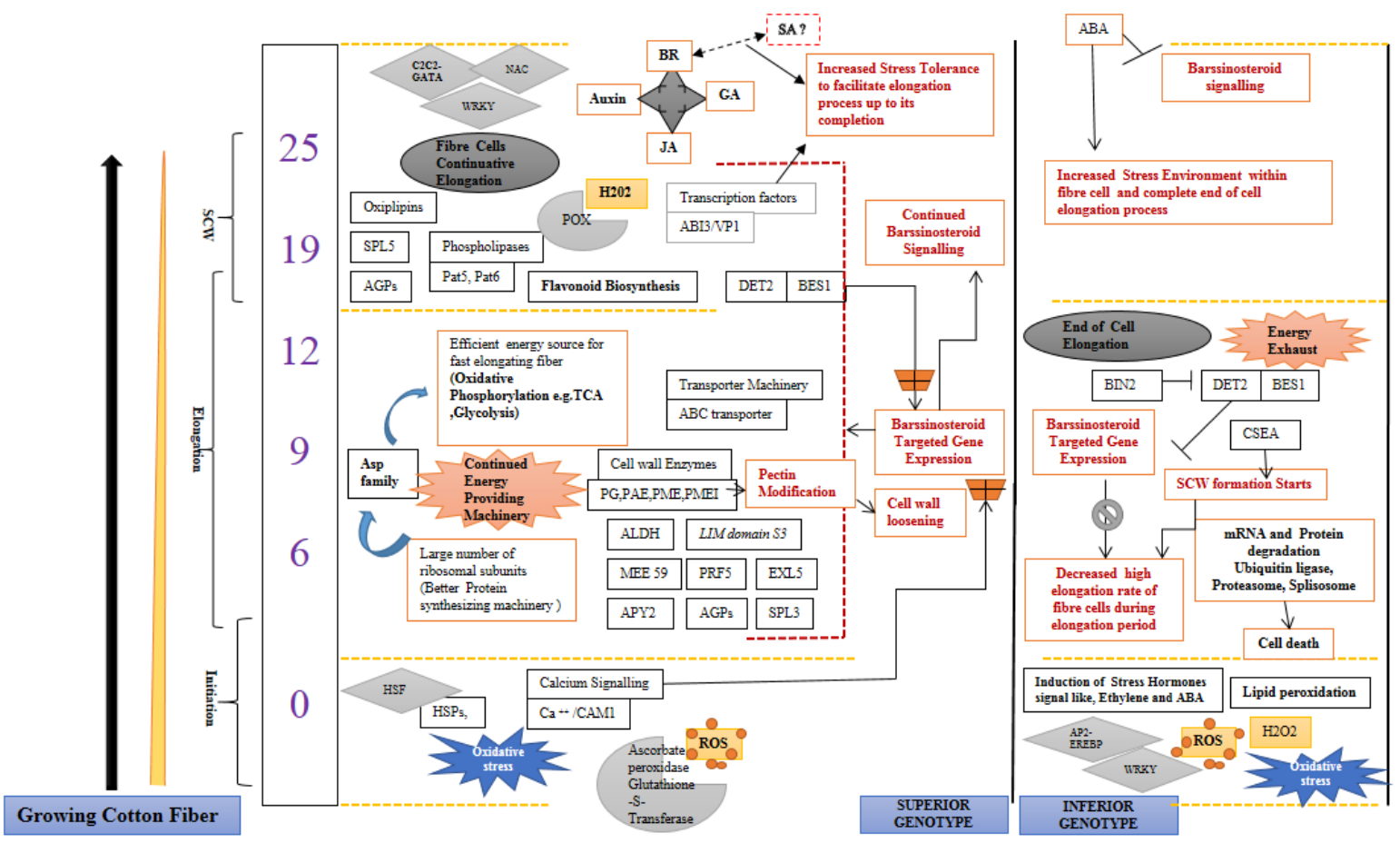

Figure 8: A hypothetical regulatory model based over-represented genes in BRTF's target genes in Superior and Inferior genotype of Gossypiumhirsutumat three developmental stages of fiber (initaion elongation and SCW stage ). This model is also supported by the previous our work (Nigam D et. Al 2013). The central black line in the model separates unique processes/genes in superior and inferior at three stages of fiber development. Various biological functions/genes involved early ( 0 to $6 \mathrm{dpa}$ ) in fiber development are shown, including genes that encode proteins involved in oxidative stress, redox signaling, and hormone signaling. At 6 to $12 \mathrm{dpa}$ in superior genotype, oxidative phosphorylation is shown as prominent processes for energy production and brassinostroid biosynthesis where BES1 and DET are positive regulator. At 19 dpa in superior genotype, peroxidase is up-regulated. Whereas in inferior genotype at early ( 0 to $6 \mathrm{dpa}$ ), oxidative stress (ROS) and stress hormone signalling suggest earlier onset of programmed cell death-like processes with exhaustive use of energy. By 6 to $12 \mathrm{dpa}$ dpa, additional processes are shown related to mRNA degradation and programmed cell death signals. At 12 to $25 \mathrm{dpa}$, cellulose synthase and pectinesterase are shown as up-regulated, leading to early cell wall maturation and cessation of elongation. 
Citation: Nigam D (2014) Integration of Brassinosteroid Signal Transduction with the Transcription Network for Fiber Development and Drought Stress in Gossypium hirsutum L. J Comput Sci Syst Biol 7: 108-114. doi:10.4172/jcsb.1000145

on gene expression and ultimately on specific plant phenotype. To systematically investigate BES1-regulated transcription downstream of BR signalling, we performed in-silico analysis identification of total 8 BRTF's target genes during cotton fiber development. We identified 3636 target genes among them large number of BES1/BZR1 target genes have been identified correlated with previous BR study in Arabidopsis; but the functions of many have not been established. An in depth understanding of the BR transcriptional network requires a combination of genetic, genomic and computational modeling approaches.

\section{Acknowledgement}

Deepti $\mathrm{N}$ is grateful to the Council of Scientific and Industrial Research, Government of India, New Millennium Technology Leadership Initiative (NMITLI) program for the financial support. Deepti $\mathrm{N}$ is thankful to CSIR for senior research fellowship. Deepti $N$ is also thanks to Dr. Samir. Sawant, Principal Scientist for providing the lab facility in conducting this research work.

\section{Conflict of Interest}

The authors declare that there is no conflict of interest.

\section{References}

1. DeLanghe EA. Lint development. Cotton physiology 1986:325-349.

2. Sun Y, Fokar M, Asami T, Yoshida S, Allen RD (2004) Characterization of the brassinosteroid insensitive 1 genes of cotton. Plant Mol Biol 54: 221-232.

3. Mandava NB (1988) Plant growth-promoting brassinosteroids. Annual Review of plant Physiology and Plant Molecular Biology 39:23-52.

4. Mayumi K, Shibaoka H (1995) A possible double role for brassinolide in the reorientation of cortical microtubules in the epidermal cells of azuki bean epicotyls. Plant and Cell Physiology 36:173-181.

5. Muñoz FJ, Labrador E, Dopico B (1998) Brassinolides promote the expression of a new Cicerarietinum beta-tubulin gene involved in the epicotyl elongation. Plant Mol Biol 37: 807-817.

6. Zurek DM, Rayle DL, McMorris TC, Clouse SD (1994) Investigation of Gene Expression, Growth Kinetics, and Wall Extensibility during BrassinosteroidRegulated Stem Elongation. Plant Physiol 104: 505-513.

7. Goda H, Sawa S, Asami T, Fujioka S, Shimada Y, et al. (2004) Comprehensive comparison of auxin-regulated and brassinosteroid-regulated genes in Arabidopsis. Plant Physiol 134: 1555-1573.

8. Goda H, Shimada Y, Asami T, Fujioka S, Yoshida S (2002) Microarray analysis of brassinosteroid-regulated genes in Arabidopsis. Plant Physiol 130: 13191334.

9. Ji SJ, Lu YC, Feng JX, Wei G, Li J, et al. (2003) Isolation and analyses of genes preferentially expressed during early cotton fiber development by subtractive PCR and cDNA array. Nucleic Acids Res 31: 2534-2543.

10. Smart LB, Vojdani F, Maeshima M, Wilkins TA (1998) Genes involved in osmoregulation during turgor-driven cell expansion of developing cotton fibersis differentially regulated. Plant Physiol 116: 1539-1549.

11. Yin Y, Vafeados D, Tao Y, Yoshida S, Asami T, et al. (2005) A new class of transcription factors mediates brassinosteroid-regulated gene expression in Arabidopsis. Cell 120: 249-259.

12. Yu X, Li L, Zola J, Aluru M, Ye H, et al. (2011) Abrassinosteroid transcriptional network revealed by genome-wide identification of BESI target genes in Arabidopsis thaliana. Plant J 65: 634-646.

13. Basso K, Margolin AA, Stolovitzky G, Klein U, Dalla-Favera R, et al. (2005) Reverse engineering of regulatory networks in human B cells. Nat Genet 37 : 382-390
14. Margolin AA, Nemenman I, Basso K, Wiggins C, Stolovitzky G, et al. (2006) ARACNE: an algorithm for the reconstruction of gene regulatory networks in a mammalian cellular context. BMC Bioinformatics 7 Suppl 1: S7.

15. Ali B, Hasan S, Hayat S, Hayat Q, Yadav S, et al. (2008) A role for brassinosteroids in the amelioration of aluminium stress through antioxidant system in mung bean ( $<$ i>Vignaradiata L. Wilczek). Environmental and Experimental Botany 62:153-159.

16. Nigam D, Kavita P, Tripathi RK, Ranjan A, Goel R, et al. (2014) Transcriptome dynamics during fibre development in contrasting genotypes of Gossypiumhirsutum L. Plant Biotechnol J 12: 204-218.

17. Bajguz A, Hayat S (2009) Effects of brassinosteroids on the plant responses to environmental stresses. Plant Physiol Biochem 47: 1-8.

18. Hayat Q, Hayat S, Irfan M, Ahmad A (2010) Effect of exogenous salicylic acid under changing environment: a review. Environmental and Experimental Botany 68:14-25.

19. Müssig C, Fischer S, Altmann T (2002) Brassinosteroid-regulated gene expression. Plant Physiol 129: 1241-1251.

20. Szekeres M, Németh K, Koncz-Kálmán Z, Mathur J, Kauschmann A et al. (1996) Brassinosteroids rescue the deficiency of CYP90, a cytochrome P450 controlling cell elongation and de-etiolation in Arabidopsis. Cell 85: 171-182.

21. Vardhini BV, Rao SSR (2003) Amelioration of osmotic stress by brassinosteroids on seed germination and seedling growth of three varieties of sorghum. Plant Growth Regulation 41:25-31.

22. Pustovoitova TN, Zhdanova NE, Zholkevich, VN (2001) Influence of epibrassinolide on adaptation processes in Cucumussativus L. plants in drought soil conditions. $6^{\text {th }}$ Conference on Regulators of Plant Growth and Development in Biotechnology, Moscow.

23. Upreti K, Murti G (2004) Effects of Brassmosteroids on Growth, Nodulation, Phytohormone Content and Nitrogenase Activity in French Bean Under Water Stress. Biologia Plantarum 48: 407-411.

24. Vert G, Nemhauser JL, Geldner N, Hong F, Chory J (2005) Molecular mechanisms of steroid hormone signaling in plants. Annu Rev Cell Dev Biol 21: 177-201.

25. Gilbert MK, John MB, Jay MS, Heping Cao,Doug JH, et al. (2013) A Transcript Profiling Approach Reveals an Abscisic Acid-Specific Glycosyltransferase (UGT73C14) Induced in Developing Fiber of Ligon lintless-2 Mutant of Cotton (Gossypiumhirsutum L.) PLoS ONE 8: e75268. 